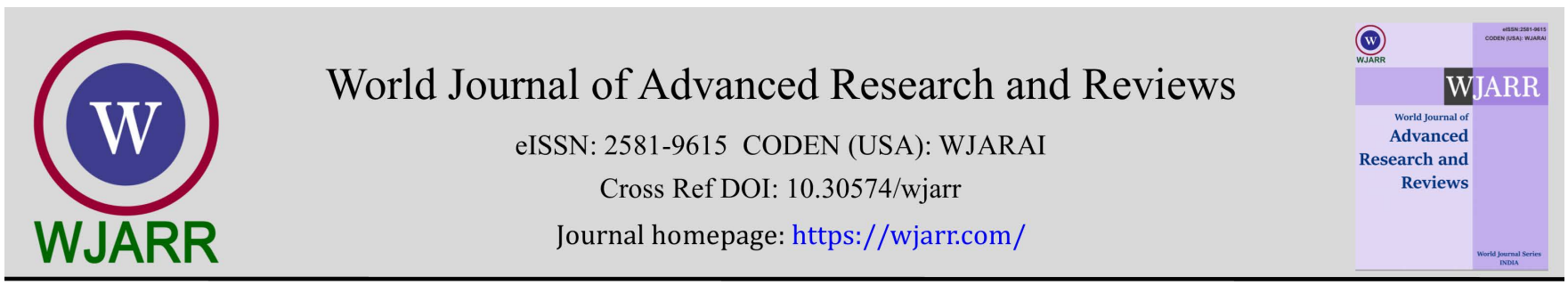

(REVIEW ARTICLE)

Check for updates

\title{
Facts and horizons of genetically modified organisms/foods and health issues
}

\author{
Rakhad Abdulrazak Alrawi 1, ${ }^{*}$ and Rafal Abdulrazak Al-rawi 2 \\ ${ }^{1}$ Grand Canyon University and Arizona College. Arizona, USA. \\ 2 Department of Pathology, Hawler Medical University, Erbil, Kurdistan, Iraq.
}

World Journal of Advanced Research and Reviews, 2022, 13(02), 071-075

Publication history: Received on 08 December 2021; revised on 10 January 2022; accepted on 12 January 2022

Article DOI: https://doi.org/10.30574/wjarr.2022.13.2.0040

\begin{abstract}
A GMO stands for genetically modified organism, whose genetic material has been artificially manipulated in a laboratory through genetic engineering. This creates plant, animal, bacteria, and virus genes that do not occur in nature. GMO foods (GMF) were introduced in the 1990s. Nowadays, GMF includes many plants and animals, whose genetic materials (DNA) structure has been modified. A biologist researcher creates GMOs by introducing genetic material, or DNA, from a different organism through a process called genetic engineering. Crops are most often genetically modified to increase production, improve diseases protection, improve resistance to insects, and herbs as well as modified to contain extra nutrients, such as vitamins and proteins. Animals can be genetically modified to increase production and improve quality. Foods produced from GMOs are often referred to as GM foods (GMF), where they are carefully studied before they are sold to the public to ensure they are safe. These studies showed that GMO foods are as healthful and safe as their non-GMO foods. There is some controversy over the benefits and risks of GMO foods. It is vital issue to discuss of benefits and risks of GMO foods, taking into account their potential effects on human health and the environment. The present article focuses on GMO plants and animal products (GMF) that may concern human consumers.
\end{abstract}

Keywords: GMO; Food; Human health; Cancer risk; Nutritional value; Crops.

\section{GMO Plants and Animals}

Researchers designed plants using genetically modified organisms, to be tougher, more nutritious, or taste better. However, people have concerns over their safety, and there is much debate about using GMOs. Many GMO plants have been modified to improve their nutritional value. An example is GMO soybeans with healthier oils that can be used to replace oils that can converted to Transfats (an unhealthy substance that is formed during hydrogenation, a process that converts unsaturated liquid oils into solid saturated fats with an extended shelf life). Research indicated that they are just as safe as non-GMO soybeans.

Genetic modification can make crops more resistant to diseases as they grow. Manufacturers use genetic modification to give foods desirable traits, as well as making crops more nutritious or enhance flavor. Crop protection is the main rationale behind this type of genetic modification. Plants that are more resistant to diseases resulted in higher yields for farmers and a more attractive product.

Additionally, research showed that GMO plants (soybeans, and corn) fed to farm animals are as safe as non-GMO animal feeds. In addition, most packaged foods contain ingredients derived from corn, soy, canola, and sugar beet. The vast majority of those crops grown in North America are genetically modified. In addition, many currently available GMO foods (GMFS) besides crops are fruit and vegetables.

\footnotetext{
${ }^{*}$ Corresponding author: Alrawi Rakhad

Grand Canyon University and Arizona College. Arizona, USA.
}

Copyright (C) 2022 Author(s) retain the copyright of this article. This article is published under the terms of the Creative Commons Attribution Liscense 4.0. 
It was indicated that there is no a connection between whether a food is a GMO or non-GMO food and antibiotics or steroids. As GMO foods do not contain more antibiotic or steroid residues than the non-GMO foods. Furthermore, processed crop derivatives and that derived from other forms of genetic engineering, such as synthetic biology including: hydrolyzed vegetable protein corn syrup, molasses, sucrose, textured vegetable protein, flavorings, vitaminsyeast products, microbes and enzymes, flavors, oils and fats, proteins, and sweeteners. The National Bioengineered Food suggested and disclosed Standard labels as "derived from bioengineering" or "bioengineered." Under the new rules, all foods containing genetically engineered ingredients should be labeled. Moreover, The Food and Drugs Administration (FDA) have determined that a GMO food should be labeled as such if it is "materially different" to its conventional counterpart. For example:

- a GMO canola oil with more lauric acid than traditional canola oil will be labeled "laurate canola oil",

- a GMO soybean oil with more oleic acid than non-GMO soybean oil must be labeled "high-oleic soybean oil",

- a GMO soybean oil with a high level of stearidonic acid, which does not naturally occur in the oil, must be labeled "stearidonate soybean oil".

The U.S. Department of Agriculture has reported that 94 percent of soybean and 91 percent of cotton crops were genetically modified by 2014. Currently, up to 90 percent of domestic corn acres are made from herbicide-tolerant seeds. Furthermore, potatoes, squash, apples, and papayas are also commonly modified. Also most GMO crops become ingredients in other foods. These include:

- $\quad$ corn starch in soups and sauces

- $\quad$ corn syrup used as a sweetener

- corn, canola, and soybean oils in mayonnaise, dressings, and breads

- $\quad$ sugar derived from sugar beets

Genetically engineered crops have been harvested for millennia. Retaining seeds from bumper crops is a time-honored agricultural tradition. Shoppers demand high-quality, pristine-looking fruits and vegetables, and wholesalers, distributors, traders, and retail sellers all demand that fresh products have a long shelf-life and spoil slowly. Consumers will not tolerate product blemishes, bruises, or minor defects when purchasing. Genetically modified crops consistently produce such items and improve the quantity and quality of harvests, safely and reliably feeding the public. Consequently, farmers resort to genetically modified seeds for harvests that provide abundant GMFs yields. Most GMFSs are not consumed directly by the public; some $90 \%$ are targeted to industrial use, from which some food derivatives are made. An example is maize. Most maize is used for the industrial production of ethanol (as gasohol), the rest being diverted to manufacture other edibles such as fructose. Fructose is widely used as a sweetener in many manufactured foods and drinks.

No known deleterious health or ecological effects have emanated from the commercialization of genetically modified crops: "There is broad scientific consensus that genetically engineered crops currently on the market are safe to eat. After 14 years of cultivation and a cumulative total of 2 billion acres planted, no adverse health or environmental effects have resulted from commercialization of genetically engineered crops. Millions of people have been eating GMFs, progressing and thriving in health for decades. Genetically engineered foods contribute enormously to the food supply and have stabilized markets while providing ample nutrition for all.

Animals can be genetically modified to produce lower-fat meat, resist certain diseases, or create less waste. Foods produced from or using GMOs are often referred to as GM foods. GMO plants may influenced livestock, apiculture, and aquaculture products because genetically engineered ingredients are common in animal feed. This influences animal products such as eggs, milk, meat, honey, and seafood [1-9].

\subsection{Health Issues}

GM foods undergo safety assessments that focus on the mutation of the genes, possible toxic properties, and nutritional effects. The main health issues of concern are:

- Allergenicity, or the potential to provoke an allergic reaction. When GM crops, such as corn or wheat, are used in the creation of other foods (corn tortillas or bread), there is a chance that consumption of these foods could cause a reaction. In one case, a pest-repelling protein used on corn to destroy insects and was only approved for animal 
feeding, was cross-pollinated with unmodified crops, and therefore entered the human supply. People with an allergy to the protein experienced reactions as severe as anaphylactic shock.

- Gene transfer,or the transfer of genes from GM foods to cells of the body or to bacteria in the gastrointestinal tract. Although the probability of gene transfer is low, a transferred gene can cause adverse health effects.

- Migration of genes from GM plants into conventional crops, and the mixing of GM and non-GM crops. Few cases have been reported where low levels of GM crops approved for animal feed were detected in products for human consumption.

- $\quad$ Environmental impact, the impacts of GMOs on the environment were studied, the results of research indicated that more than $80 \%$ of all genetically modified crops grown worldwide have been engineered for herbicide tolerance. As a result, the use of toxic herbicides, such as Roundup (key ingredient is glyphosate) has increased fifteen-fold since GMOs were first introduced. In March 2015, the World Health Organization determined that the glyphosate in Roundup is probably carcinogenic to humans.

- Antibacterial resistance, there is concern that genetic modification, which can boost a crop's resistance to disease or make it more tolerant to herbs, could affect the ability of people to defend against illness. There is a small chance that the genes in food can transfer to cells the body or bacteria in the gut. Some GMO plants contain genes that make them resistant to certain antibiotics. This resistance could pass on to humans. There is growing concern globally that people are becoming increasingly resistant to antibiotics. There is a chance that GMO foods could be contributing to this crisis. The WHO have said that the risk of gene transfer is low. As a precaution, however, it has set guidelines for the manufacturers of GMO foods.

- Allergic reactions, some people believe that GMO foods have more potential to trigger allergic reactions. This is because they may contain genes from an allergen (a food that prompts an allergic reaction). It is worth noting that there have been no reports of allergic effects of any GMO foods currently on the market. Not unless one allergic to the non-GMO version of that food. Most food allergies (90\%) are caused by allergens found in just eight foods: peanuts, tree nuts, milk, eggs, wheat, soy, shellfish, and fish. For example, soy is an allergenic food available in a GMO version. If you are allergic to foods made with traditional (non-GMO) soy, you will be allergic to foods made with GMO soy. If one does not allergic to foods made with traditional soy, he does not be allergic to foods made with GMO soy. Research shows that GMO foods are no more likely to cause allergies than non-GMOs. The way GMOs are created allows scientists to know precisely which new proteins are produced in a plant. Scientists perform tests to make sure these proteins are not allergens. This type of testing, called allergenicity testing, is always part of the process for developing GMOs. In addition, FDA regulations ensure that foods derived from GMOs must be as safe as the non-GMOs you eat. Some people wonder if the cases of celiac disease are rising because of GMOs. Celiac disease is a serious condition that affects the digestive system. It is caused by an abnormal sensitivity to gluten, a protein found in wheat, rye, and barley. There is no evidence that GMOs on the market today cause celiac disease. In addition, there is no GMO wheat, rye, or barley for commercial sale in the United States.

\subsection{Cancer risk}

As genetically engineering foods is a relatively new practice, little is known about the long-term effects and safety and the main health issues associated with GMO foods are debated. Research showed that GMO crops are not changed in ways that would increase the risk of cancer for the humans or animals that eat them. Results revealed that cancer rates among peoples were not connected with eating GMOs. Although some cancers can be prevented because their precipitating factors or triggering agents are known (for example, the human papilloma viruses, asbestos, alcohol, and smoking), most causes of cancer are unknown. Once neoplastic change is established, there is no absolute cure other than total surgical removal or total replacement of the cancerous cells (in leukemias and lymphomas). Chemotherapy and radiation therapy are essentially life-prolonging treatments, but rarely total cures. The recent report claiming that GMFss are causally associated with cancer development in rats has been debunked by informed opinion: genetically tumourprone rats were used; a spurious construct and research protocol was followed; and the statistical approach used did not satisfy confounding factors. The publication was apparently not subject to satisfactory objective refereeing, and certain tainted financial interests were also operative. All the foregoing factors skewed the results, rendering them invalid and not significant.Eating fresh foods is preferable to eating processed foods, and fresh GMF (or the nutritional derivatives from GMF) are regularly and globally eaten in vast quantities without any proven side effects. Industrialized countries have been successful in producing ample sustenance for their populations from GMF. All manufactured edibles with a long shelf life, such as canned foods, soda pop, snack food, and other preserved comestibles, may have traces of carcinogenic substances that contribute to or facilitate carcinogenesis. Among the many compounds 
implicated are bisphenol A in the plastic linings of metal cans, benzopyrenes in barbecue, nitrites in delicatessen meats, carbon dioxide gas in soda pop, and saccharin in diet foods. These molecules are not derived from GMF. All are doserelated, and all are acknowledged to be cancer promoters in abusively high doses.Promoting and enhancing vaccination strategies helps to reduce virally induced cancers. Universal screening by health care workers assists in hastening detection, diagnosis, and therapy, with successful outcomes [7, $9-13]$.

\section{Conclusion}

Avoiding GMF will neither stop nor prevent carcinogenesis. Healthy eating from modern mass food production demands informed choice to realize the full benefits of nutrition and to eschew co-factors for cancer. Vaccination against known causes is desirable, and complementary therapies help to palliate cancer morbidity. Scientists are free to express their ideas, but they bear a responsibility to be objective and to provide a full, open, rational, and transparent account of any research evidence procured to substantiate their views. While there, is limited scientific evidence demonstrating that eating organic food can decrease cancer risk, what is the evidence regarding its counterpart, GMOs? For now, there is insufficient evidence to conclude that consuming GMF can increase cancer risk. In the absence of credible independent long-term feeding studies, the safety of GMF is unknown. Sixty-four countries around the world, including Australia, Japan, and all of the countries in the European Union, require genetically modified foods to be labeled. Canada does not require any GMF labeling. GMF are not currently labeled in the United States. However, the National Bioengineered Food Disclosure Standard (NBFDS) was published in the Federal Register on December 21, 2018. This law, which you may have heard called the DARK Act, is the start of mandatory GMF labeling in the United States. It means that some (but not all) products containing GMF will have to be labeled by 2022. In its current form, categorical exemptions prevent this law from delivering the meaningful protections Americans deserve. There is insufficient evidence to conclude that GMO and/or GMF can increase cancer risk. Eating more fruits and vegetables, regardless of how they are grown, is more beneficial than avoiding any potential risks of pesticides used on fruits and vegetables. Some researchers believe that eating GMF can contribute to the development of cancer. They argue that because the disease is caused by mutations in DNA, it is dangerous to introduce new genes into the body. The American Cancer Society (ACS) has announced that there is no evidence for this. However, they note that no evidence of harm is not the same as proof of safety and that reaching a conclusion will require more research.

Humans have selectively bred animals and plants since time immemorial, manipulating genes to obtain benefits. The process was slow, taking many generations until recently, when genomics and biotechnology accelerated genetic mutation processes. Understanding speedy gene modification allows for vigorous species propagation, with crop yields increased in quality and quantity through genetic engineering.

Recently, animal research has suggested that genetically modified foods (GMF) are causally related to carcinogenesis. Among the many implications of that research is the notion that when GMF are avoided no cancer will develop, and a cancer cure is procurable through prophylactic dietary selection. Alternative medical practices are replete with myths of unproven cures, most promulgated as definitive medicine ${ }^{3}$. Modern therapy has changed some cancers into chronic diseases, and cancer survival rates have improved over recent decades. Yet some cancers, such as pancreatic and liver cancer, still have short survival durations and poor prognosis. Contrary to established data, some well-documented aggressive cases of cancer have entered into total remission, confirmed through histopathology of biopsy samples. However, such events are extremely rare.

Therefore, the take home message is that long-term impacts of most GMOs are unknown.

\section{Compliance with ethical standards}

\section{Acknowledgments}

The authors thank Mr. Abdulaziz Abdulhameed Abdulla for his help and support during the period of the preparing the article.

\section{Disclosure of conflict of interest}

Authors declare that they have no competing interest. 


\section{References}

[1] Fernandez-Cornejo J, Seth J W. USDA ERS - Adoption of Genetically Engineered Crops in the U.S. Recent Trends in GE Adoption. . United States Department of Agriculture, Economic Research Service. 2015.

[2] Mortensen DA, Egan JF, Maxwell BD, Ryan MR, Smith RG. Navigating a critical juncture for sustainable weed management. BioScience. 2012; 62(1):75-84.

[3] FDA.https://www.fda.gov/food/food-new-plant-varieties/understanding- new-plant-varieties.

[4] FDA.https://www.nas-sites.org/ge-crops/2016/05/17/report/

[5] FDA.https://www.ghr.nlm.nih.gov/condition/celiac-disease.

[6] Kloor K. No, genetically modified corn won't give you cancer. National Post. 2012; A11.

[7] Del Fabbro E, Bruera E, Demark-Whanefried W, Bowling T, Hopkinson JB, Baracos VE, editors. Nutrition and the Cancer Patient. New York, NY: Oxford University Press. 2010.

[8] Ronald P. Scientific American. 2011. Genetically engineered crops-what, how and why.

[9] Touyz LZG. Politics, health, and mesothelioma: when it comes to cancer, none is one too many. Curr Oncol.2012;19:e374-5.

[10] De Vendômois JS, Roullier F, Cellier D,Séralini GE. A comparison of the effects of three GM corn varieties on mammalian health. Int J Biol Sci. 2009;5: 706-26.

[11] Kustin ME. Glyphosate is Spreading Like a Cancer Across the U.S. EWG. Environmental Working Group, 07 Apr. 2015.

[12] Touyz LZG. Genetically modified foods, cancer, and diet: myths and reality. CurrOncol. 2013 Apr; 20(2): e59-e61.

[13] Dana-Farber Cancer Institute. Is There Evidence That GMOs Can Cause Cancer? 2019. 